\title{
Moments of volumes of lower-dimensional random simplices are not monotone
}

\author{
Benjamin Reichenwallner ${ }^{1}$ (D)
}

Received: 20 February 2017 / Accepted: 15 June 2017 / Published online: 21 June 2017

C The Author(s) 2017. This article is an open access publication

\begin{abstract}
In a $d$-dimensional convex body $K$, for $n \leq d+1$, random points $X_{0}, \ldots, X_{n-1}$ are chosen according to the uniform distribution in $K$. Their convex hull is a random $(n-1)$-simplex with probability 1 . We denote its $(n-1)$-dimensional volume by $V_{K[n]}$. The $k$-th moment of the $(n-1)$-dimensional volume of a random $(n-1)$-simplex is monotone under set inclusion, if $K \subseteq L$ implies that the $k$-th moment of $V_{K[n]}$ is not larger than that of $V_{L[n]}$. Extending work of Rademacher (Mathematika 58:77-91, 2012) and Reichenwallner and Reitzner (Mathematika 62:949-958, 2016), it is shown that for $n \leq d$, the moments of $V_{K[n]}$ are not monotone under set inclusion. As a consequence, the nonmonotonicity of the expected surface area of the convex hull of $n \leq d+1$ uniform random points in a $d$-dimensional convex body follows.
\end{abstract}

Keywords Random polytopes - Random simplices - Approximation of convex bodies $\cdot$ Sylvester's problem $\cdot$ Monotonicity under inclusion

Mathematics Subject Classification Primary 60D05 · Secondary 52A22

\section{Introduction}

For a $d$-dimensional convex body $K$ and $n \leq d+1$, we denote the ( $n-1)$-dimensional volume of the convex hull of $n$ independently and uniformly distributed random points $X_{0}, \ldots, X_{n-1}$ in $K$ by $V_{K[n]}$. Since the $n$ points are not contained in any $(n-2)$ -

Communicated by A. Constantin.

$\triangle$ Benjamin Reichenwallner

benjamin.reichenwallner@sbg.ac.at

1 Fachbereich Mathematik, Universität Salzburg, Hellbrunnerstraße 34, 5020 Salzburg, Austria 
dimensional hyperplane with probability 1 , their convex hull is almost surely an $(n-1)$-simplex. In 2006, Meckes [5] asked whether for a pair of convex bodies $K, L \subseteq \mathbb{R}^{d}, K \subseteq L$ would imply

$$
\mathbb{E} V_{K[d+1]} \leq \mathbb{E} V_{L[d+1]}
$$

Interest in this question comes from the fact that it would imply a positive solution to the slicing problem, see e.g. [7]. For a more general statement of the conjecture we refer to [9].

In 2012, Rademacher [7] showed that Meckes' conjecture (1) is not true in general, since there exist counterexamples for each dimension $d \geq 4$. His results were generalized to higher moments of $V_{K[d+1]}$ by Reichenwallner and Reitzner [8], and the expected value in dimension three was handled in [4]:

Theorem 1 Let $k \in \mathbb{N}$ and $d \geq 2$. For two-dimensional convex bodies $K$ and $L$, $K \subseteq L$ implies that $\mathbb{E} V_{K[d+1]} \leq \mathbb{E} V_{L[d+1]}$ and $\mathbb{E} V_{K[d+1]}^{2} \leq \mathbb{E} V_{L[d+1]}^{2}$. Unless $d=2$ and $k \in\{1,2\}$, there exist two $d$-dimensional convex bodies $K, L$ satisfying $K \subseteq L$ and $\mathbb{E} V_{K[d+1]}^{k}>\mathbb{E} V_{L[d+1]}^{k}$.

Note that the problem is trivial in dimension one. In this paper, we perform another extension by considering convex hulls of $n \leq d+1$ random points in $d$-dimensional convex bodies. In order to keep the statement of the result simple, we restrict to $n \leq d$ and refer to the theorem above for the case $n=d+1$.

Theorem 2 Let $d, n, k \in \mathbb{N}$ with $d \geq 2$ and $2 \leq n \leq d$. Then there exist two $d$-dimensional convex bodies $K, L$ satisfying $K \subseteq \bar{L}$ and $\mathbb{E} V_{K[n]}^{k}>\mathbb{E} V_{L[n]}^{k}$.

Due to Rademacher [7], it is already known that the moments of $V_{K[n]}$ are not monotone under set inclusion in general. However, it might still come as a surprise that for $n \leq d$, there exist counterexamples for any moment in any dimension, particularly in dimension two, where the first two moments of the area of a random triangle are monotone.

Theorem 1 is shown using an equivalence statement, first stated in Lemma 12 in [7]. In consideration of Proposition 16 ibidem, it is clear that the lemma can be generalized in the following way:

Lemma 1 For $d, n, k \in \mathbb{N}$ with $2 \leq n \leq d+1$, monotonicity under inclusion of the map $K \mapsto \mathbb{E} V_{K[n]}^{k}$, where $K$ ranges over all d-dimensional convex bodies, holds if and only if we have for each convex body $K \subseteq \mathbb{R}^{d}$ and for each $x \in$ bd $K$ that $\mathbb{E} V_{K[n]}^{k} \leq \mathbb{E} V_{K[n], x}^{k}$

Here, $V_{K[n], x}$ denotes the $(n-1)$-dimensional volume of a random $(n-1)$-simplex, which is the convex hull of a fixed point $x$ and $n-1$ independently and uniformly distributed random points in $K$. The lemma allows us to consider one convex body $K$, rather than a pair of convex bodies, and compute two different moments: the moment of the $(n-1)$-dimensional volume of a random $(n-1)$-simplex in $K$ as well as the same, but fixing one of the $n$ points to be a point on the boundary of $K$, denoted by bd $K$. 
For the case $n=d+1$, Rademacher chooses $K$ to be a $d$-dimensional halfball and $x$ the midpoint of the base which is a $(d-1)$-dimensional ball. This pair forms the counterexample in Theorem 1 to the monotonicity of all moments for $d \geq 4$, and to the monotonicity of the $k$-th moment for $k \geq 11$ in dimension two and for $k \geq 2$ in dimension three (see [8]; in fact, in dimension two, it does so for $k \geq 8$ ). Additional counterexamples are given by the triangle, together with the midpoint of one of its edges, for $d=2$ and $k \geq 3$ in [8] and the tetrahedron, together with the centroid of one of its facets, for $d=3$ and $k=1$ in [4].

For our generalization, we use the following lemma, which allows us to create counterexamples inductively:

Lemma 2 Let $d, n, k \in \mathbb{N}$ with $2 \leq n \leq d+1$. If there exist two $d$-dimensional convex bodies $K, L$ satisfying $K \subseteq L$ and $\mathbb{E} V_{K[n]}^{k}>\mathbb{E} V_{L[n]}^{k}$, there also exist two $(d+1)$-dimensional convex bodies $K^{\prime}, L^{\prime}$ satisfying $K^{\prime} \subseteq L^{\prime}$ and $\mathbb{E} V_{K^{\prime}[n]}^{k}>\mathbb{E} V_{L^{\prime}[n]}^{k}$.

We use the counterexamples mentioned above as well as Lemma 2 to get results on the monotonicity of the moments of $V_{K[n]}$, where $K$ ranges over all $d$-dimensional convex bodies, $d \geq 2$ and $2 \leq n \leq d$. Since, obviously, monotonicity holds in dimension 1, in order to show that the moments of the length of a random chord in $d$-dimensional convex bodies are not monotone for $d>1$, we create another counterexample by computing the moments of the length of a random chord inside a rectangular triangle where both points are randomly chosen as well as the same where one point is fixed to be the midpoint of the hypotenuse.

Proposition 1 For the triangle $T_{2}$ with vertices $(0,0),(1,0)$ and $(0,1)$, and $c_{2}=$ $(1 / 2,1 / 2)$ the midpoint of the edge $\{(x, y): x, y \geq 0, x+y=1\}$, we have that $\mathbb{E} V_{T_{2}[2], c_{2}}^{k}<\mathbb{E} V_{T_{2}[2]}^{k}$ for each $k \in \mathbb{N}$.

Concerning the case $n=3, k=1$, we need to construct a pair of three-dimensional convex bodies, or one three-dimensional convex body and a suitable point on its boundary, leading to a counterexample. Already the computation of the expected volume of a random tetrahedron in dimension three is a hard problem, and the same is supposed to be true for the expected area of a random triangle in dimension three.

The only three-dimensional convex sets where the expected volume of a random full-dimensional simplex is known are the ball [6], the cube [12] and the tetrahedron [1], and the ball is the only one for which the expected area of a random triangle inside is known. Since numerical simulations suggest that the ball cannot form a counterexample in our concerns, and neither can the cube, we consider random triangles in a tetrahedron, which nothing is known about. A suitable choice for $x$ should be the center $c$ of one of the facets, but we have to take into account that the considered moments depend on the shape of $T$ as well as of the facet which $c$ lies in. Additionally, already the determination of the expected volume of a random tetrahedron in a tetrahedron $T$, done by Buchta and Reitzner [1], was extremely hard, and it seems that a computation of $\mathbb{E} V_{T[3]}$ and $\mathbb{E} V_{T[3], c}$ is out of reach. Neverless we will prove the following proposition.

Proposition 2 For the tetrahedron $T_{3}$ with vertices $(0,0,0),(1,0,0),(0,1,0)$ and $(0,0,1)$, and $c_{3}=(1 / 3,1 / 3,1 / 3)$ the centroid of the facet $\{(x, y, z): x, y, z \geq$ $0, x+y+z=1\}$, we have that $\mathbb{E} V_{T_{3}[3], c_{3}}<\mathbb{E} V_{T_{3}[3]}$. 
A combination of this result and a direct computation of $\mathbb{E} V_{T_{3}[3]}^{2}$ and $\mathbb{E} V_{T_{3}[3], c_{3}}^{2}$ with Rademacher's Lemma 1 yields Theorem 2. Proposition 2 is obtained by a combination of methods from stochastic geometry with results from approximation theory. In the background, we use a result about the precise approximation of the square root function by suitable polynomials.

A next step might naturally be given by a generalization of Theorems 1 and 2 to intrinsic volumes, among them the surface area. While a derivation of results on its higher moments seems to be rather involved, it appears that the nonmonotonicity of the expected surface area of a random simplex, eventually not of full dimension, is a direct consequence of Theorem 2 . For a $d$-dimensional convex body $K$ and $2 \leq n \leq d+1$, let $S_{K[n]}$ denote the surface area of the convex hull of $n$ points that are chosen according to uniform distribution in $K$. Since the (relative) boundary of an $(n-1)$-simplex is given by the union of $n$ facets and the expected value is additive, the following corollary holds true:

Corollary 1 Let $d, n \in \mathbb{N}$ with $d \geq 2$ and $2 \leq n \leq d+1$. Then there exist two $d$-dimensional convex bodies $K, L$ satisfying $K \subseteq L$ and $\mathbb{E} S_{K[n]}>\mathbb{E} S_{L[n]}$.

More generally, it shall be mentioned that the expected value of the sum of the $m$-dimensional volumes of all $m$-faces of the convex hull of $n \geq m+2$ uniform random points in a convex body $K$ of dimension $d \geq n-1$ is not monotone under set inclusion.

Finally, we consider a similar problem: Assume that $n$ points are uniformly distributed on the boundary of a convex body $K$ and let $\mathcal{V}_{K[n]}$ be the $(n-1)$-dimensional volume of their convex hull. Then we can use a technique similar to that in the background of Lemma 2 to show that, in general, monotonicity under set inclusion does not hold for the moments of $(n-1)$-dimensional volumes of this type of random $(n-1)$-simplices either. In detail, we show the following:

Theorem 3 Let $d, n, k \in \mathbb{N}$ with $d \geq 3$ and $2 \leq n \leq d$. Unless $d=3, n=3$ and $k \in\{1,2\}$, there exist two $d$-dimensional convex bodies $K, L$ satisfying $K \subseteq L$ and $\mathbb{E} \mathcal{V}_{K[n]}^{k}>\mathbb{E} \mathcal{V}_{L[n]}^{k}$.

This paper is organized in the following way. We prove Lemma 2 in Sect. 2. In Sect. 3, we compute the moments of the lengths of two types of random chords inside a triangle as described above, and thereafter we give bounds for the expected areas of random triangles in a tetrahedron. These results will be used in Sect. 5 for the proof of Theorem 2. Finally, Theorem 3 will be proved in Sect. 6.

As a general reference for the tools and results we need in the following, we refer to the book on Stochastic and Integral Geometry by Schneider and Weil [10]. More recent surveys on random polytopes are due to Hug [3] and Reitzner [9].

\section{Proof of Lemma 2}

Let $d, n, k \in \mathbb{N}$ with $2 \leq n \leq d+1$ and assume that there exist two $d$-dimensional convex bodies $K$ and $L$ satisfying $K \subseteq L$ and $\mathbb{E} V_{K[n]}^{k}>\mathbb{E} V_{L[n]}^{k}$. Our goal is to 
construct two $(d+1)$-dimensional convex bodies $K^{\prime}, L^{\prime}$ satisfying similar conditions. For $\varepsilon>0$, we define two $(d+1)$-dimensional convex bodies by

$$
K_{\varepsilon}:=K \times[0, \varepsilon], \quad L_{\varepsilon}:=L \times[0, \varepsilon] .
$$

Then, $K_{\varepsilon} \subseteq L_{\varepsilon}$ for all $\varepsilon$, and if $\mathbb{E} V_{K_{\varepsilon}[n]}^{k}$ and $\mathbb{E} V_{L_{\varepsilon}[n]}^{k}$ converge to $\mathbb{E} V_{K[n]}^{k}$ and $\mathbb{E} V_{L[n]}^{k}$, resp., for $\varepsilon$ tending to zero, there exists an $\varepsilon_{0}>0$ such that $\mathbb{E} V_{K_{\varepsilon_{0}}[n]}^{k}>\mathbb{E} V_{L_{\varepsilon_{0}}[n]}^{k}$.

It remains to proof the continuity of the map $\varepsilon \mapsto \mathbb{E} V_{K_{\varepsilon}[n]}^{k}$ in $\varepsilon=0$. We express the $(n-1)$-dimensional volume of an $(n-1)$-simplex with vertices $x_{0}, \ldots, x_{n-1}$ using a matrix that contains the vectors spanning the $(n-1)$-simplex in its columns:

$$
\begin{aligned}
& \operatorname{vol}_{n-1} \operatorname{conv}\left(x_{0}, x_{1}, \ldots, x_{n-1}\right) \\
& \quad=\frac{1}{(n-1) !} \sqrt{\operatorname{det}\left(\left(x_{1}-x_{0}, \ldots, x_{n-1}-x_{0}\right)^{T}\left(x_{1}-x_{0}, \ldots, x_{n-1}-x_{0}\right)\right)} .
\end{aligned}
$$

Then we have for $\varepsilon>0$ :

$$
\begin{aligned}
& \mathbb{E} V_{K_{\varepsilon}[n]}^{k}=\frac{1}{\operatorname{vol} K_{\varepsilon}^{n}} \int_{K_{\varepsilon}^{n}} \operatorname{vol}_{n-1} \operatorname{conv}\left(x_{0}, \ldots, x_{n-1}\right)^{k} d\left(x_{0}, \ldots, x_{n-1}\right) \\
& =\frac{1}{\varepsilon^{n} \operatorname{vol}_{d} K^{n}} \frac{1}{((n-1) !)^{k}} \int_{K^{n}} \int_{[0, \varepsilon]^{n}} \operatorname{det} B^{k / 2} d\left(z_{0}, \ldots, z_{n-1}\right) d\left(x_{0}, \ldots, x_{n-1}\right),
\end{aligned}
$$

where $B=\left(b_{i j}\right)_{1 \leq i, j \leq n-1}$ with

$$
b_{i j}=\sum_{m=1}^{d}\left(x_{i}^{(m)}-x_{0}^{(m)}\right)\left(x_{j}^{(m)}-x_{0}^{(m)}\right)+\left(z_{i}-z_{0}\right)\left(z_{j}-z_{0}\right),
$$

writing $x_{i}=\left(x_{i}^{(1)}, \ldots, x_{i}^{(d)}, z_{i}\right)$. Now, fix the first $d$ coordinates of the points $x_{0}, \ldots, x_{n-1}$ and let $\zeta:=\min _{z_{i}} \operatorname{det} B$ and $Z:=\max _{z_{i}} \operatorname{det} B$, where the minimum and the maximum are taken over all possible choices of $\left(z_{0}, \ldots, z_{n-1}\right) \in[0, \varepsilon]^{n}$. The extrema exist since the determinant is continuous and the intervall $[0, \varepsilon]$ is compact. We have:

$$
\begin{aligned}
& \frac{1}{\operatorname{vol}_{d} K^{n}} \frac{1}{((n-1) !)^{k}} \int_{K^{n}} \zeta^{k / 2} d\left(x_{0}, \ldots, x_{n-1}\right) \leq \mathbb{E} V_{K_{\varepsilon}[n]}^{k} \\
& \leq \frac{1}{\operatorname{vol}_{d} K^{n}} \frac{1}{((n-1) !)^{k}} \int_{K^{n}} Z^{k / 2} d\left(x_{0}, \ldots, x_{n-1}\right) .
\end{aligned}
$$

If $\varepsilon$ tends to zero, the coordinates $z_{i}$ do so as well, and all summands of $\zeta$ and $Z$ containing a factor $z_{i}$ vanish in the limit. Therefore, we get that

$$
\mathbb{E} V_{K[n]}^{k} \leq \lim _{\varepsilon \rightarrow 0} \mathbb{E} V_{K_{\varepsilon}[n]}^{k} \leq \mathbb{E} V_{K[n]}^{k},
$$

which implies that $\lim _{\varepsilon \rightarrow 0} \mathbb{E} V_{K_{\varepsilon}[n]}^{k}=\mathbb{E} V_{K[n]}^{k}$. 


\section{Random chords in a triangle}

Essential for our investigations of the case $n=2$ are the moments of two different types of random distances in a given triangle $T$. In detail, we need the moments of the length of a random chord in a right-angled triangle, which arises as the segment between two uniformly chosen random points in this triangle, as well as the moments of the distance of a random point in this triangle from the midpoint of its hypotenuse, which is equivalent to the length of a random chord where one point is fixed to be this midpoint.

\subsection{Moments of the distance of a random point from a point on the boundary}

We first prove a lemma that will be useful for the computation of the moments of the distance of a random point from a fixed point on the boundary, since we can dissect a triangle by a line through this fixed point.

Lemma 3 Let $T$ be a triangle with vertices $A, B$ and $C$. Denote the lengths of the edges opposite to $A, B$ and $C$ by $a, b$ and $c$, resp., and the angles in these corners by $\alpha, \beta$ and $\gamma$, resp. Then it holds for the moments of the distance of a random point in $T$ from $A$ :

$$
\begin{aligned}
\mathbb{E} V_{T[2], A}^{k}= & \frac{2(c \sin \beta)^{k+1}}{(k+2) a}\left(\cos \beta_{2} F_{1}\left(\frac{1}{2}, \frac{k+3}{2} ; \frac{3}{2} ; \cos \beta^{2}\right)\right. \\
& \left.-\cos (\alpha+\beta)_{2} F_{1}\left(\frac{1}{2}, \frac{k+3}{2} ; \frac{3}{2} ; \cos (\alpha+\beta)^{2}\right)\right) .
\end{aligned}
$$

Here, the function

$$
{ }_{2} F_{1}\left(a_{1}, a_{2} ; b ; x\right)=\sum_{k=0}^{\infty} \frac{\left(a_{1}\right)_{k}\left(a_{2}\right)_{k}}{(b)_{k}} \frac{x^{k}}{k !}
$$

with $(a)_{k}=a(a+1) \cdots(a+k-1)$ is a hypergeometric function.

Proof Since the expected length of a random chord in a triangle does not depend on the position of the latter, we build our coordinate system ensuring that $A$ is identical to the origin $o$ and the edge $c$ lies on the $x$-axis. Then:

$$
\mathbb{E} V_{T[2], o}^{k}=\frac{1}{\operatorname{vol} T} \int_{T}\|x\|^{k} d x .
$$

Note that $\operatorname{vol} T=a c \sin \beta / 2$. Using polar coordinates, we get that

$$
\mathbb{E} V_{T[2], o}^{k}=\frac{2}{a c \sin \beta} \int_{0}^{\alpha} \int_{0}^{l(\varphi)} r^{k+1} d r d \varphi=\frac{1}{k+2} \frac{2}{a c \sin \beta} \int_{0}^{\alpha} l(\varphi)^{k+2} d \varphi,
$$


where $l(\varphi)$ denotes the length of the intersection of the triangle with the line whose angle to the $x$-axis is $\varphi$. By the sine rules, $l(\varphi)=c \sin \beta / \sin (\beta+\varphi)$, and

$$
\mathbb{E} V_{T[2], o}^{k}=\frac{2(c \sin \beta)^{k+1}}{(k+2) a} \int_{0}^{\alpha} \frac{1}{\sin (\beta+\varphi)^{k+2}} d \varphi .
$$

With

$$
\int \frac{1}{\sin \varphi^{k+2}} d \varphi=-\cos \varphi_{2} F_{1}\left(\frac{1}{2}, \frac{k+3}{2} ; \frac{3}{2} ; \cos \varphi^{2}\right)
$$

we get that

$$
\begin{aligned}
\mathbb{E} V_{T[2], o}^{k}= & \frac{2(c \sin \beta)^{k+1}}{(k+2) a}\left(\cos \beta_{2} F_{1}\left(\frac{1}{2}, \frac{k+3}{2} ; \frac{3}{2} ; \cos \beta^{2}\right)\right. \\
& \left.-\cos (\alpha+\beta)_{2} F_{1}\left(\frac{1}{2}, \frac{k+3}{2} ; \frac{3}{2} ; \cos (\alpha+\beta)^{2}\right)\right) .
\end{aligned}
$$

Given this lemma, we can compute the moments of the distance of a random point from a point on the boundary of a triangle.

Proposition 3 Let $T$ be a triangle with vertices $A, B$ and $C$. Denote the lengths of the edges opposite to $A, B$ and $C$ by $a, b$ and $c$, resp., and the angles in these corners by $\alpha, \beta$ and $\gamma$, resp. Furthermore, let $D$ be a point lying on the edge between $A$ and $B$ and $c_{1}$ the distance of $D$ from $A$. Then it holds for the moments of the distance of a random point in $T$ from $D$ :

$$
\begin{aligned}
\mathbb{E} V_{T[2], D}^{k}= & \frac{2}{(k+2) b c \sin \alpha}\left(\left(c_{1} \sin \alpha\right)^{k+2} \cos \alpha_{2} F_{1}\left(\frac{1}{2}, \frac{k+3}{2} ; \frac{3}{2} ; \cos \alpha^{2}\right)\right. \\
& -\left(c_{1} \sin \alpha\right)^{k+2} \cos (\alpha+\delta)_{2} F_{1}\left(\frac{1}{2}, \frac{k+3}{2} ; \frac{3}{2} ; \cos (\alpha+\delta)^{2}\right) \\
& +\left(\left(c-c_{1}\right) \sin \beta\right)^{k+2} \cos \beta_{2} F_{1}\left(\frac{1}{2}, \frac{k+3}{2} ; \frac{3}{2} ; \cos \beta^{2}\right) \\
& \left.+\left(\left(c-c_{1}\right) \sin \beta\right)^{k+2} \cos (\beta-\delta)_{2} F_{1}\left(\frac{1}{2}, \frac{k+3}{2} ; \frac{3}{2} ; \cos (\beta-\delta)^{2}\right)\right),
\end{aligned}
$$

where

$$
\delta=\arcsin \left(b \sin \alpha / \sqrt{c_{1}^{2}+b^{2}-2 c_{1} b \cos \alpha}\right) .
$$

Proof We split $T$ into two parts by the line segment between $C$ and $D$. Denote the length of this line segment by $d$ and the angle between $A D$ and $D C$ by $\delta$. Then, by cosine and sine rule, 


$$
d=\sqrt{c_{1}^{2}+b^{2}-2 c_{1} b \cos \alpha} \text { and } \delta=\arcsin (b \sin \alpha / d)
$$

If we denote the triangle $A C D$ by $T_{1}$ and the triangle $B C D$ by $T_{2}$, we have:

$$
\mathbb{E} V_{T[2], D}^{k}=\frac{\operatorname{vol} T_{1}}{\operatorname{vol} T} \mathbb{E} V_{T_{1}[2], D}^{k}+\frac{\operatorname{vol} T_{2}}{\operatorname{vol} T} \mathbb{E} V_{T_{2}[2], D}^{k}
$$

The previous lemma yields the result.

In order to find a counterexample to the monotonicity of the moments of the length of random chords, it suffices to consider one specific triangle. We choose a right-angled, isosceles one and apply Proposition 3 to this triangle.

Corollary 2 For the triangle $T_{2}$ with vertices $(0,0),(0,1)$ and $(1,0)$, and $c_{2}=$ $(1 / 2,1 / 2)$, we have:

$$
\mathbb{E} V_{T_{2}[2], c_{2}}^{k}=\frac{2^{1 / 2-k}}{k+2}{ }_{2} F_{1}\left(\frac{1}{2}, \frac{k+3}{2} ; \frac{3}{2} ; \frac{1}{2}\right) .
$$

We give some values of $\mathbb{E} V_{T_{2}[2], c_{2}}^{k}$ for this specific triangle:

$$
\begin{aligned}
& \mathbb{E} V_{T_{2}[2], c_{2}}=\frac{1}{6 \sqrt{2}}(2+\sqrt{2} \operatorname{arcsinh} 1)=0.3825 \ldots, \\
& \mathbb{E} V_{T_{2}[2], c_{2}}^{2}=\frac{1}{6}=0.1666 \ldots, \\
& \mathbb{E} V_{T_{2}[2], c_{2}}^{3}=\frac{1}{160 \sqrt{2}}(14+3 \sqrt{2} \operatorname{arcsinh} 1)=0.0783 \ldots, \\
& \mathbb{E} V_{T_{2}[2], c_{2}}^{4}=\frac{7}{180}=0.0388 \ldots
\end{aligned}
$$

\subsection{Moments of the length of a random chord}

In this subsection, we complete our investigations of the triangle by a computation of the moments of the length of a random chord in a triangle, where both endpoints of the chord are uniformly distributed points in the triangle.

Proposition 4 Let $T$ be a triangle with vertices $E_{1}, E_{2}$ and $E_{3}$. Denote the length of the edge opposite to $E_{i}$ by $e_{i}$ and the angle in this corner by $\eta_{i}, i=1,2,3$. Then it holds for the moments of the distance of two random point in $T$ :

$$
\begin{aligned}
\mathbb{E} V_{T[2]}^{k}= & \frac{8}{(k+2)(k+3)(k+4)} \frac{1}{\left(e_{1} e_{3} \sin \eta_{2}\right)^{2}} \sum_{i \neq j} \sin \eta_{i}^{k+3} e_{j}^{k+4} \\
& \times\left[\cos \eta_{i} \cos \eta_{j 2} F_{1}\left(\frac{1}{2}, \frac{k+3}{2} ; \frac{3}{2} ; \cos \eta_{j}^{2}\right)\right.
\end{aligned}
$$




$$
\begin{aligned}
& +\cos \eta_{i}^{2}{ }_{2} F_{1}\left(\frac{1}{2}, \frac{k+3}{2} ; \frac{3}{2} ; \cos \eta_{i}^{2}\right) \\
& \left.+\frac{\sin \eta_{i}}{k+2}\left(\frac{1}{\sin \eta_{j}^{k+2}}-\frac{1}{\sin \eta_{i}^{k+2}}\right)\right] .
\end{aligned}
$$

Proof As before, the area of the triangle is given by $e_{1} e_{3} \sin \eta_{2} / 2$. Using the affine Blaschke-Petkantschin formula—see e.g. [10]—we integrate over all lines $H$ intersecting the triangle:

$$
\begin{aligned}
\mathbb{E} V_{T[2]}^{k} & =\frac{1}{\operatorname{vol} T^{2}} \int_{T^{2}}\left\|x_{0}-x_{1}\right\|^{k} d\left(x_{0}, x_{1}\right) \\
& =\frac{4}{\left(e_{1} e_{3} \sin \eta_{2}\right)^{2}} \int_{A(2,1)} \int_{(H \cap T)^{2}}\left\|x_{0}-x_{1}\right\|^{k} d\left(x_{0}, x_{1}\right) d H,
\end{aligned}
$$

where $A(2,1)$ denotes the affine Grassmannian of lines in $\mathbb{R}^{2}$. We represent a line $H$ by its unit normal vector $u \in S_{1}$ and its distance $t>0$ from the origin and we therefore denote the line by

$$
H_{t, u}=\left\{x \in \mathbb{R}^{2}:\langle x, u\rangle=t\right\} .
$$

We choose the normalization of the Haar measure $d H$ in such a way that $d H=$ $d t d u$, where $d t$ and $d u$ correspond to Lebesgue measures in $\mathbb{R}$ and $S_{1}$. We write the appearing integral as an expectation to get

$$
\begin{aligned}
\mathbb{E} V_{T[2]}^{k} & =\frac{4}{\left(e_{1} e_{3} \sin \eta_{2}\right)^{2}} \int_{S_{1}} \int_{0}^{\infty} \int_{\left(H_{t, u} \cap T\right)^{2}}\left\|x_{0}-x_{1}\right\|^{k+1} d\left(x_{0}, x_{1}\right) d t d u \\
& =\frac{4}{\left(e_{1} e_{3} \sin \eta_{2}\right)^{2}} \int_{S_{1}} \int_{0}^{\infty} \operatorname{vol}\left(H_{t, u} \cap T\right)^{2} \mathbb{E} V_{H_{t, u} \cap T}^{k+1} d t d u .
\end{aligned}
$$

The $(k+1)$-st moment of the distance of two random points in an interval $I$ of length $l$ has already been computed by Solomon [11], amongst others, to be given by

$$
\mathbb{E} V_{I}^{k+1}=\frac{2 l^{k+1}}{(k+2)(k+3)} \text {. }
$$

Hence, we obtain

$$
\mathbb{E} V_{T[2]}^{k}=\frac{8}{(k+2)(k+3)} \frac{1}{\left(e_{1} e_{3} \sin \eta_{2}\right)^{2}} \int_{S_{1}} \int_{0}^{\infty} \operatorname{vol}\left(H_{t, u} \cap T\right)^{k+3} d t d u
$$


A line $H_{t, u}$ that intersects the triangle $T$ a.s. meets exactly two edges of $T$. It splits $T$ into a triangle and a quadrangle. We say that $H_{t, u}$ cuts off a vertex $E_{i}$ from $T$ if $E_{i}$ is contained in the triangular part. Furthermore, we write

$$
\mathcal{I}^{(i)}=\int_{S_{1}} \int_{0}^{\infty} \mathbb{1}\left(H_{t, u} \text { cuts off } E_{i} \text { from } T\right) \operatorname{vol}\left(H_{t, u} \cap T\right)^{k+3} d t d u
$$

for $i=1,2,3$, which gives

$$
\mathbb{E} V_{T[2]}^{k}=\frac{8}{(k+2)(k+3)} \frac{1}{\left(e_{1} e_{3} \sin \eta_{2}\right)^{2}}\left(\mathcal{I}^{(1)}+\mathcal{I}^{(2)}+\mathcal{I}^{(3)}\right) .
$$

We state the following lemma, which will be proved right after the end of the proof of this proposition:

Lemma 4 It holds for $i=1,2,3$ :

$$
\begin{aligned}
\mathcal{I}^{(i)}= & \frac{\sin \eta_{i}^{k+3}}{k+4}\left(e _ { j } ^ { k + 4 } \left[\cos \eta_{i} \cos \eta_{j}{ }_{2} F_{1}\left(\frac{1}{2}, \frac{k+3}{2} ; \frac{3}{2} ; \cos \eta_{j}^{2}\right)\right.\right. \\
& +\cos \eta_{i}^{2}{ }_{2} F_{1}\left(\frac{1}{2}, \frac{k+3}{2} ; \frac{3}{2} ; \cos \eta_{i}^{2}\right) \\
& \left.+\frac{\sin \eta_{i}}{k+2}\left(\frac{1}{\sin \eta_{j}^{k+2}}-\frac{1}{\sin \eta_{i}^{k+2}}\right)\right] \\
& +e_{l}^{k+4}\left[\cos \eta_{i} \cos \eta_{l} F_{1}\left(\frac{1}{2}, \frac{k+3}{2} ; \frac{3}{2} ; \cos \eta_{l}^{2}\right)\right. \\
& +\cos \eta_{i}^{2}{ }_{2} F_{1}\left(\frac{1}{2}, \frac{k+3}{2} ; \frac{3}{2} ; \cos \eta_{i}^{2}\right) \\
& \left.\left.+\frac{\sin \eta_{i}}{k+2}\left(\frac{1}{\sin \eta_{l}^{k+2}}-\frac{1}{\sin \eta_{i}^{k+2}}\right)\right]\right)
\end{aligned}
$$

This lemma immediately yields the result.

Proof of Lemma 4 Let $i \in\{1,2,3\}$. We substitute $u=(\cos \varphi, \sin \varphi)$ such that the line $\varphi=0$ contains the edge between $E_{i}$ and $E_{j}$ for $j \neq i$ fixed, and by the well-known fact that $d u=d \varphi$ and with $H_{t, \varphi}=\left\{(x, y) \in \mathbb{R}^{2}: x \cos \varphi+y \sin \varphi=t\right\}$, we get the integral

$$
\mathcal{I}^{(i)}=\int_{-\pi}^{\pi} \int_{0}^{\infty} \mathbb{1}\left(H_{t, \varphi} \text { cuts off } E_{i} \text { from } T\right) \operatorname{vol}\left(H_{t, \varphi} \cap T\right)^{k+3} d t d \varphi
$$

Let $l \in\{1,2,3\} \backslash\{i, j\}$. A line $H_{t, \varphi}$ cuts off $E_{i}$ from $T$ if and only if $\varphi \in\left[\eta_{i}-\right.$ $\pi / 2, \pi / 2]$ and 
(i) $0 \leq t \leq e_{j} \cos \left(\eta_{1}-\varphi\right)$, if $\varphi \leq \frac{\pi}{2}-\eta_{j}$,

(ii) $0 \leq t \leq e_{l} \cos \varphi$, if $\varphi \geq \frac{\pi}{2}-\eta_{j}$.

We use the notation $l(t, \varphi)=\operatorname{vol}\left(H_{t, \varphi} \cap T\right)$ for the length of the intersection of $T$ with a line $H_{t, \varphi}$. Basic trigonometric considerations show that

$$
l(t, \varphi)=t\left(\tan \left(\eta_{i}-\varphi\right)+\tan \varphi\right)=t \frac{\sin \eta_{i}}{\cos \varphi \cos \left(\eta_{i}-\varphi\right)} .
$$

This leads to the expression

$$
\begin{aligned}
\mathcal{I}^{(i)} & =\int_{\eta_{i}-\pi / 2}^{\pi / 2-\eta_{j}}\left(\frac{\sin \eta_{i}}{\cos \varphi \cos \left(\eta_{i}-\varphi\right)}\right)^{k+3} \int_{0}^{e_{j} \cos \left(\eta_{i}-\varphi\right)} t^{k+3} d t d \varphi \\
& +\int_{\pi / 2-\eta_{j}}^{\pi / 2}\left(\frac{\sin \eta_{i}}{\cos \varphi \cos \left(\eta_{i}-\varphi\right)}\right)^{k+3} \int_{0}^{e_{l} \cos \varphi} t^{k+3} d t d \varphi \\
& =\frac{\sin \eta_{i}^{k+3}}{k+4}\left(e_{j}^{k+4} \int_{\eta_{i}-\pi / 2}^{\pi / 2-\eta_{j}} \frac{\cos \left(\eta_{i}-\varphi\right)}{\cos \varphi^{k+3}} d \varphi+e_{l}^{k+4} \int_{\pi / 2-\eta_{j}}^{\pi / 2} \frac{\cos \varphi}{\cos \left(\eta_{i}-\varphi\right)^{k+3}} d \varphi\right) .
\end{aligned}
$$

With a substitution $\psi=\eta_{i}-\varphi$ and with $\cos \left(\eta_{i}-\varphi\right)=\cos \eta_{i} \cos \varphi+\sin \eta_{i} \sin \varphi$, we can compute the outer integral:

$$
\begin{aligned}
\mathcal{I}^{(i)}= & \frac{\sin \eta_{i}^{k+3}}{k+4}\left(e _ { j } ^ { k + 4 } \left[\cos \eta_{i} \cos \eta_{j}{ }_{2} F_{1}\left(\frac{1}{2}, \frac{k+3}{2} ; \frac{3}{2} ; \cos \eta_{j}^{2}\right)\right.\right. \\
& +\cos \eta_{i}^{2}{ }_{2} F_{1}\left(\frac{1}{2}, \frac{k+3}{2} ; \frac{3}{2} ; \cos \eta_{i}^{2}\right) \\
& \left.+\frac{\sin \eta_{i}}{k+2}\left(\frac{1}{\sin \eta_{j}^{k+2}}-\frac{1}{\sin \eta_{i}^{k+2}}\right)\right] \\
& +e_{l}^{k+4}\left[\cos \eta_{i} \cos \eta_{l} F_{1}\left(\frac{1}{2}, \frac{k+3}{2} ; \frac{3}{2} ; \cos \eta_{l}^{2}\right)\right. \\
& +\cos \eta_{i}^{2}{ }_{2} F_{1}\left(\frac{1}{2}, \frac{k+3}{2} ; \frac{3}{2} ; \cos \eta_{i}^{2}\right) \\
& \left.\left.+\frac{\sin \eta_{i}}{k+2}\left(\frac{1}{\sin \eta_{l}^{k+2}}-\frac{1}{\sin \eta_{i}^{k+2}}\right)\right]\right) .
\end{aligned}
$$

Again, we apply this proposition to the right-angled, isosceles triangle. 
Corollary 3 For the triangle $T_{2}$ with vertices $(0,0),(0,1)$ and $(1,0)$, we have:

$$
\mathbb{E} V_{T_{2}[2]}^{k}=\frac{2^{(5-k) / 2}+2^{7 / 2}}{(k+2)(k+3)(k+4)}{ }_{2} F_{1}\left(\frac{1}{2}, \frac{k+3}{2} ; \frac{3}{2} ; \frac{1}{2}\right) \text {. }
$$

As before, we give some values of $\mathbb{E} V_{T_{2}[2]}^{k}$ for this triangle:

$$
\begin{aligned}
& \mathbb{E} V_{T_{2}[2]}=\frac{1+2 \sqrt{2}}{30}(2+\sqrt{2} \operatorname{arcsinh} 1)=0.4142 \ldots, \\
& \mathbb{E} V_{T_{2}[2]}^{2}=\frac{2}{9}=0.2222 \ldots, \\
& \mathbb{E} V_{T_{2}[2]}^{3}=\frac{1+4 \sqrt{2}}{840}(14+3 \sqrt{2} \operatorname{arcsinh} 1)=0.1405 \ldots, \\
& \mathbb{E} V_{T_{2}[2]}^{4}=\frac{1}{10}=0.1 .
\end{aligned}
$$

\section{Random triangles in a tetrahedron}

In this section, our aim is to show that for a specific tetrahedron $T$ and the center $c$ of one of its facets, we have

$$
\mathbb{E} V_{T[3]}>\mathbb{E} V_{T[3], c}
$$

Therefore, we want to find polynomials $P(x)=\sum_{i=0}^{n} a_{i} x^{i}$ and $Q(x)=\sum_{i=0}^{l} b_{i} x^{i}$ such that $P(x) \leq|x|$ and $Q(x) \geq|x|$ for relevant ranges of values $x \in \mathbb{R}$ and consequently

$$
\mathbb{E} V_{T[3]} \geq \mathbb{E} P\left(V_{T[3]}\right)>\mathbb{E} Q\left(V_{T[3], c}\right) \geq \mathbb{E} V_{T[3], c},
$$

which suffices in order to show that the pair $(T, c)$ is a counterexample to monotonicity of the expected area of a random triangle in dimension three. Simulations suggest that

$$
\mathbb{E} V_{T[3]}=0.0592 \ldots \text { and } \mathbb{E} V_{T[3], c}=0.0466 \ldots
$$

and conjecture (2) should hold true.

\subsection{A lower bound for the expected area of a random triangle in a tetrahedron}

Let $T$ be a tetrahedron. For random points $X_{0}, X_{1}, X_{2} \in T$, we write $X_{i}=\left(x_{i}, y_{i}, z_{i}\right)$. The volume of the triangle with vertices $X_{0}, X_{1}$ and $X_{2}$ is given by

$$
\begin{aligned}
V_{T[3]}= & \frac{1}{2} \sqrt{\operatorname{det}\left(\left(\begin{array}{l}
x_{1}-x_{0} y_{1}-y_{0} z_{1}-z_{0} \\
x_{2}-x_{0} y_{2}-y_{0} z_{2}-z_{0}
\end{array}\right)\left(\begin{array}{l}
x_{1}-x_{0} x_{2}-x_{0} \\
y_{1}-y_{0} y_{2}-y_{0} \\
z_{1}-z_{0} z_{2}-z_{0}
\end{array}\right)\right)} \\
& =\frac{1}{2} D\left(x_{0}, \ldots, z_{2}\right)^{1 / 2},
\end{aligned}
$$


and this is the square root of a polynomial of degree four in the coordinates of $X_{0}, X_{1}$ and $X_{2}$. For even moments of $V_{T[3]}$, we get rid of the square root:

$$
\mathbb{E} V_{T[3]}^{2 k}=\operatorname{vol} T^{-3} 2^{-2 k} \int_{T} \int_{T} \int_{T} D\left(x_{0}, \ldots, z_{2}\right)^{k} d\left(x_{0}, y_{0}, z_{0}\right) d\left(x_{1}, y_{1}, z_{1}\right) d\left(x_{2}, y_{2}, z_{2}\right) .
$$

Note that the expectation $\mathbb{E} V_{T[3]}$ depends on the shape of the tetrahedron $T$. We consider the specific tetrahedron

$$
T_{3}=\left\{(x, y, z) \in \mathbb{R}^{3}: x, y, z \geq 0, x+y+z \leq 1\right\},
$$

i.e., that with vertices $(0,0,0),(1,0,0),(0,1,0)$ and $(0,0,1)$. Expanding the determinant, the polynomial $D$ can be written as

$$
D\left(x_{0}, \ldots, z_{2}\right)=\sum_{i=1}^{63} s_{i} \text { with } s_{i}=\varepsilon_{i} 2^{c_{i}} x_{0}^{e_{i 0}} \cdots z_{2}^{e_{i 8}}
$$

where $\varepsilon_{i} \in\{-1,1\}, c_{i} \in\{0,1\}$ and $e_{i j} \geq 0, j=0, \ldots, 8$, are explicitly given constants and $\sum_{j} e_{i j}=4$ for $i=1, \ldots, 63$. By the Multinomial Theorem, and using the multinomial coefficient

$$
\left(\begin{array}{c}
k \\
k_{1}, \ldots, k_{63}
\end{array}\right)=\frac{k !}{k_{1} ! \cdots k_{63} !}
$$

the $k$-th power of it can be rewritten as

$$
\begin{aligned}
D\left(x_{0}, \ldots, z_{2}\right)^{k} & =\sum_{\sum_{1}^{63} k_{i}=k}\left(\begin{array}{c}
k \\
k_{1}, \ldots, k_{63}
\end{array}\right) \prod_{i=1}^{63} s_{i}^{k_{i}} \\
& =\sum_{\sum_{1}^{63} k_{i}=k}(-1)^{k^{\prime}} 2^{k^{\prime \prime}}\left(\begin{array}{c}
k \\
k_{1}, \ldots, k_{63}
\end{array}\right) \prod_{i=0}^{2} x_{i}^{l_{1}} y_{i}^{m_{1}} z_{i}^{n_{1}} .
\end{aligned}
$$

For completeness, we list the exponents appearing in (5):

$$
\begin{aligned}
k^{\prime}= & k_{19}+\cdots+k_{37}+k_{40}+k_{44}+k_{47}+k_{51}+k_{54}+k_{55}+k_{59}+k_{63}, \\
k^{\prime \prime}= & k_{19}+\cdots+k_{63}, \\
l_{0}= & 2 k_{1}+\cdots+2 k_{4}+2 k_{19}+2 k_{20}+k_{27}+k_{29}+k_{33}+k_{35}+k_{37}+\cdots+k_{48}, \\
m_{0}= & 2 k_{5}+2 k_{9}+2 k_{13}+2 k_{14}+k_{21}+k_{23}+2 k_{25}+2 k_{26}+k_{34}+k_{36}+\cdots+k_{38} \\
& +k_{43}+k_{44}+k_{49}+k_{50}+k_{55}+\cdots+k_{60}, \\
n_{0}= & 2 k_{7}+2 k_{11}+2 k_{15}+2 k_{17}+k_{22}+k_{24}+k_{28}+k_{30}+2 k_{31}+2 k_{32}+k_{40}+k_{41} \\
& +k_{46}+k_{47}+k_{52}+k_{53}+k_{55}+k_{56}+k_{58}+k_{59}+k_{61}+k_{62},
\end{aligned}
$$




$$
\begin{aligned}
l_{1}= & 2 k_{5}+\cdots+2 k_{8}+2 k_{21}+2 k_{22}+k_{25}+k_{29}+k_{31}+k_{35}+k_{37}+\cdots+k_{42} \\
& +k_{49}+\cdots+k_{54}, \\
m_{1}= & 2 k_{1}+2 k_{10}+2 k_{15}+2 k_{16}+k_{19}+k_{23}+2 k_{27}+2 k_{28}+k_{32}+k_{36}+k_{37}+k_{39} \\
& +k_{43}+k_{45}+k_{49}+k_{51}+k_{55}+\cdots+k_{57}+k_{61}+\cdots+k_{63}, \\
n_{1}= & 2 k_{3}+2 k_{12}+2 k_{13}+2 k_{18}+k_{20}+k_{24}+k_{26}+k_{30}+2 k_{33}+2 k_{34}+k_{40}+k_{42} \\
& +k_{46}+k_{48}+k_{52}+k_{54}+k_{55}+k_{57}+k_{58}+k_{60}+\cdots+k_{63}, \\
l_{2}= & 2 k_{9}+2 k_{10}+2 k_{11}+2 k_{12}+2 k_{23}+2 k_{24}+k_{25}+k_{27}+k_{31}+k_{33}+k_{43} \\
& +\cdots+k_{54}, \\
m_{2}= & 2 k_{2}+2 k_{6}+2 k_{17}+2 k_{18}+k_{19}+k_{21}+2 k_{29}+2 k_{30}+k_{32}+k_{34}+k_{38}+k_{39} \\
& +k_{44}+k_{45}+k_{50}+k_{51}+k_{58}+\cdots+k_{63}, \\
n_{2}= & 2 k_{4}+2 k_{8}+2 k_{14}+2 k_{16}+k_{20}+k_{22}+k_{26}+k_{28}+2 k_{35}+2 k_{36}+k_{41}+k_{42} \\
& +k_{47}+k_{48}+k_{53}+k_{54}+k_{56}+k_{57}+k_{59}+k_{60}+k_{62}+k_{63} .
\end{aligned}
$$

Integration of the monomials over the tetrahedron $T_{3}$ gives with the substitution $z=t, y=s(1-t), x=r(1-s)(1-t)$ that

$$
\begin{aligned}
\int_{T_{3}} x^{l_{i}} y^{m_{i}} z^{n_{i}} d(x, y, z) & =\int_{0}^{1} r^{l_{i}} d r \int_{0}^{1} s^{m_{i}}(1-s)^{l_{i}+1} d s \int_{0}^{1} t^{n_{i}}(1-t)^{l_{i}+m_{i}+2} d t \\
& =\frac{1}{l_{i}+1} B\left(m_{i}+1, l_{i}+2\right) B\left(n_{i}+1, l_{i}+m_{i}+3\right) \\
& =\frac{l_{i} ! m_{i} ! n_{i} !}{\left(l_{i}+m_{i}+n_{i}+3\right) !}
\end{aligned}
$$

Combining this with Eqs. (4) and (5) gives

$$
\mathbb{E} V_{T_{3}[3]}^{2 k}=6^{3} 2^{-2 k} \sum_{\sum_{1}^{63} k_{i}=k}(-1)^{k^{\prime}} 2^{k^{\prime \prime}}\left(\begin{array}{c}
k \\
k_{1}, \ldots, k_{63}
\end{array}\right) \prod_{i=0}^{2} \frac{l_{i} ! m_{i} ! n_{i} !}{\left(l_{i}+m_{i}+n_{i}+3\right) !}
$$

We list the first five even moments of the area of a random triangle in our tetrahedron $T_{3}$ :

$$
\begin{aligned}
& \mathbb{E} V_{T_{3}[3]}^{2}=\frac{9}{1600}=5.625 \cdot 10^{-3}, \\
& \mathbb{E} V_{T_{3}[3]}^{4}=\frac{27}{196000} \approx 1.37755 \cdot 10^{-4}, \\
& \mathbb{E} V_{T_{3}[3]}^{6}=\frac{3161}{379330560} \approx 8.3331 \cdot 10^{-6}, \\
& \mathbb{E} V_{T_{3}[3]}^{8}=\frac{93957}{106247680000} \approx 8.8432 \cdot 10^{-7},
\end{aligned}
$$




$$
\mathbb{E} V_{T_{3}[3]}^{10}=\frac{209022679}{1551386124288000} \approx 1.34733 \cdot 10^{-7} .
$$

We have seen that the ( $2 k)$-th moment of $V_{T_{3}[3]}$ can be computed, with fast increasing complexity in $k$. Also note that the area of a triangle in $T_{3}$ is not larger than $\sqrt{3} / 2$, which is the area of the facet $\left\{(x, y, z) \in \mathbb{R}^{3}: x, y, z \geq 0, x+y+z=1\right\}$. Hence, we want to approximate the square root function in the interval $[0,3 / 4]$ by a polynomial

$$
P(x)=\sum_{i=0}^{n} a_{i} x^{i}
$$

of degree $n$ for some $n \in \mathbb{N}$ such that $P(x) \leq \sqrt{x}$ for all $x \in\left[0, \frac{3}{4}\right]$. This way, we can approximate $\mathbb{E} V_{T_{3}[3]}$ from below by the polynomial $P$ :

$$
\mathbb{E} V_{T_{3}[3]} \geq \mathbb{E} P\left(V_{T_{3}[3]}\right) \text {. }
$$

Moreover, the best polynomial for fixed $n \in \mathbb{N}$ can be found via the linear optimization problem

$$
\max _{P} \mathbb{E} P\left(V_{T_{3}[3]}\right)=\max _{a_{i}} \sum_{i=0}^{n} a_{i} \mathbb{E} P\left(V_{T_{3}[3]}\right)^{2 i} \quad \text { s.t. } \quad P\left(x^{2}\right) \leq x, x \in\left[0, \frac{\sqrt{3}}{2}\right] .
$$

This constraint is infinite dimensional, but we get an upper bound on $\mathbb{E} P\left(V_{T_{3}[3]}\right)$ via the finite dimensional linear program

$$
\begin{aligned}
\max _{P} \mathbb{E} P\left(V_{T_{3}[3]}\right)= & \max _{a_{i}} \sum_{i=0}^{n} a_{i} \mathbb{E} V_{T_{3}[3]}^{2 i} \quad \text { s.t. } P\left(x_{\ell}^{2}\right) \leq x_{\ell}, x_{\ell} \in\left[0, \frac{\sqrt{3}}{2}\right], \\
& \ell=0, \ldots, L .
\end{aligned}
$$

For $n=6$ and $L=200$ equidistant points $x_{\ell} \in\left[0, \frac{\sqrt{3}}{2}\right]$, we numerically compute via Matlab and the optimization toolbox CVX [2] that

$$
\mathbb{E} P\left(V_{T_{3}[3]}\right)<0.04647
$$

and in consideration of the simulations in (3), this yields that we do not get a sufficiently precise estimate using only $n=6$ even moments.

We solve the above linear program for $n=7$ and $L=1000$, get the squares of three interpolation nodes with the square root function in $\left(0, \frac{\sqrt{3}}{2}\right)$ and rationalize these points to

$$
\left\{x_{1}, x_{2}, x_{3}\right\}=\left\{\frac{2}{19}, \frac{4}{15}, \frac{8}{17}\right\} \text {. }
$$

Additionally, we take the points $x_{0}=0$ and $x_{4}=\frac{47}{54}>\frac{\sqrt{3}}{2}$ as interpolation nodes of our desired polynomial. We want to use these five points to get a polynomial of degree 7. Consider a variation of Lemma 2 in [4] with two conditions 


$$
P\left(x_{j}^{2}\right)=x_{j} \text { and } P^{\prime}\left(x_{j}^{2}\right)=\frac{1}{2 x_{j}}
$$

for $j=1,2,3$ and the single conditions $P\left(x_{0}^{2}\right)=P(0)=0$ and $P\left(x_{4}^{2}\right)=x_{4}$. Then we have with $f(x)=\sqrt{x}$ that

$$
f(x)-P(x)=\frac{f^{(n+1)}(\xi)}{(n+1) !} x\left(x-x_{4}^{2}\right) \prod_{j=1}^{3}\left(x-x_{j}^{2}\right)^{2} \geq 0
$$

for some $\xi \in\left[0, x_{4}^{2}\right]$, since $f^{(n+1)}(x)<0, x \geq 0$ and $x \leq x_{4}$ for all $x \in\left[0, \frac{3}{4}\right]$.

We solve the interpolation problem and finally get a polynomial $P_{\text {cert }}(x)=$ $\sum_{i=1}^{7} a_{i} x^{i}$ of degree 7 with explicitly given rational coefficients $a_{1}, \ldots, a_{7}$ and the property $P_{\text {cert }} \leq \sqrt{x}$, and this polynomial yields with usage of the even moments computed above that

$$
\begin{aligned}
& \mathbb{E} V_{T_{3}[3]} \geq \mathbb{E} P_{\text {cert }}\left(V_{T_{3}[3]}\right) \\
& =\frac{91096443868688279627145529306423486222767653883418444589}{1940591908276502542424150723573750536040094315231313920000} \\
& >0.046942 .
\end{aligned}
$$

\subsection{An upper bound for the expected area of a random triangle in a tetrahedron where one point is fixed}

Now we consider the convex hull of two random points $X_{1}, X_{2}$ and one fixed point in a tetrahedron $T$. We write again $X_{i}=\left(x_{i}, y_{i}, z_{i}\right)$ and choose the centroid $c=$ $\left(x_{c}, y_{c}, z_{c}\right)$ of one of the facets of $T$ as third point. The volume of the triangle with vertices $X_{1}, X_{2}$ and $c$ is given by

$$
\begin{aligned}
V_{T[3], c}= & \frac{1}{2} \sqrt{\operatorname{det}\left(\left(\begin{array}{l}
x_{1}-x_{c} y_{1}-y_{c} z_{1}-z_{c} \\
x_{2}-x_{c} y_{2}-y_{c} z_{2}-z_{c}
\end{array}\right)\left(\begin{array}{l}
x_{1}-x_{c} x_{2}-x_{c} \\
y_{1}-y_{c} y_{2}-y_{c} \\
z_{1}-z_{c} z_{2}-z_{c}
\end{array}\right)\right)} \\
& =\frac{1}{2} D\left(x_{0}, \ldots, z_{c}\right)^{1 / 2},
\end{aligned}
$$

and this is again the square root of a polynomial of degree four in the coordinates of $X_{1}, X_{2}$ and $c$. As before, we have:

$$
\mathbb{E} V_{T[3], c}^{2 k}=\operatorname{vol} T^{-2} 2^{-2 k} \int_{T} \int_{T} D\left(x_{1}, \ldots, z_{c}\right)^{k} d\left(x_{1}, y_{1}, z_{1}\right) d\left(x_{2}, y_{2}, z_{2}\right) .
$$

The expectation $\mathbb{E} V_{T[3], c}$ particularly depends on the facet whose centroid we choose to be the fixed vertex. We take again the tetrahedron $T_{3}$ as defined in Sect. 4.1 and choose $c_{3}=(1 / 3,1 / 3,1 / 3)$, the centroid of the facet $\left\{(x, y, z) \in \mathbb{R}^{3}: x, y, z \geq\right.$ $0, x+y+z=1\}$, to be the fixed vertex of the random triangle. 
Expanding the determinant and inserting the coordinates of $c_{3}$, the polynomial $D$ can be displayed as

$$
D\left(x_{1}, \ldots, z_{c_{3}}\right)=\frac{1}{9} \sum_{i=1}^{54} s_{i} \quad \text { with } \quad s_{i}=\varepsilon_{i} 2^{c_{i}} 3^{d_{i}} x_{1}^{e_{i 0}} \cdots z_{2}^{e_{i 5}},
$$

where $\varepsilon_{i} \in\{-1,1\}, c_{i}, d_{i} \in\{0,1,2\}$ and $e_{i j} \geq 0, j=0, \ldots, 5$, are explicitly given constants and $\sum_{j} e_{i j} \leq 4$ for $i=1, \ldots, 54$. Again with usage of the Multinomial Theorem, the series representation of the $k$-th power of it is given by

$$
\begin{aligned}
D\left(x_{1}, \ldots, z_{c_{3}}\right)^{k} & =3^{-2 k} \sum_{\sum_{1}^{54} k_{i}=k}\left(\begin{array}{c}
k \\
k_{1}, \ldots, k_{54}
\end{array}\right) \prod_{i=1}^{54} s_{i}^{k_{i}} \\
& =3^{-2 k} \sum_{\sum_{1}^{54} k_{i}=k}(-1)^{k^{\prime}} 2^{k^{\prime \prime}} 3^{k^{\prime \prime \prime}}\left(\begin{array}{c}
k \\
k_{1}, \ldots, k_{54}
\end{array}\right) \prod_{i=1}^{2} x_{i}^{l_{1}} y_{i}^{m_{1}} z_{i}^{n_{1}},
\end{aligned}
$$

where we use abbreviations analogously as in the previous proof:

$$
\begin{aligned}
k^{\prime}= & k_{7}+\cdots+k_{10}+k_{12}+k_{15}+k_{17}+k_{18}+k_{21}+\cdots+k_{33}+k_{52}+\cdots+k_{54}, \\
k^{\prime \prime}= & k_{1}+\cdots k_{6}+2 k_{7}+\cdots+2 k_{9}+k_{10}+\cdots+k_{45}+k_{52}+\cdots+k_{54}, \\
k^{\prime \prime \prime}= & k_{22}+\cdots+k_{45}+2 k_{46}+\cdots+2 k_{54}, \\
l_{1}= & 2 k_{1}+k_{7}+k_{10}+\cdots+k_{13}+2 k_{22}+2 k_{23}+k_{28}+k_{32}+k_{34}+\cdots+k_{39} \\
& +2 k_{46}+2 k_{47}+k_{52}+k_{53}, \\
m_{1}= & 2 k_{3}+k_{8}+k_{10}+k_{14}+k_{18}+k_{19}+k_{24}+2 k_{26}+2 k_{27}+k_{33}+k_{34} \\
& +k_{38}+k_{40}+k_{42}+\cdots+k_{44}+2 k_{48}+2 k_{50}+k_{52}+k_{54}, \\
n_{1}= & 2 k_{5}+k_{9}+k_{12}+k_{16}+k_{18}+k_{20}+k_{25}+k_{29}+2 k_{30}+2 k_{31}+k_{36} \\
& +k_{39}+k_{41}+k_{42}+k_{44}+k_{45}+2 k_{49}+2 k_{51}+k_{53}+k_{54}, \\
l_{2}= & 2 k_{2}+k_{7}+k_{14}+\cdots+k_{17}+2 k_{24}+2 k_{25}+k_{26}+k_{30}+k_{34} \\
& +\cdots+k_{37}+k_{40}+k_{41}+2 k_{48}+2 k_{49}+k_{52}+k_{53}, \\
m_{2}= & 2 k_{4}+k_{8}+k_{11}+k_{15}+k_{20}+\cdots+k_{22}+2 k_{28}+2 k_{29}+k_{31}+k_{35} \\
& +k_{38}+k_{40}+k_{42}+k_{43}+k_{45}+2 k_{46}+2 k_{51}+k_{52}+k_{54}, \\
n_{2}= & 2 k_{6}+k_{9}+k_{13}+k_{17}+k_{19}+k_{21}+k_{23}+k_{27}+2 k_{32}+2 k_{33}+k_{37} \\
& +k_{39}+k_{41}+k_{43}+k_{44}+k_{45}+2 k_{47}+2 k_{50}+k_{53}+k_{54} .
\end{aligned}
$$

Now, Eqs. (6), (8) and (9) give

$$
\mathbb{E} V_{T_{3}[3], c_{3}}^{2 k}=6^{2-2 k} \sum_{\sum_{1}^{54} k_{i}=k}(-1)^{k^{\prime}} 2^{k^{\prime \prime}} 3^{k^{\prime \prime \prime}}\left(\begin{array}{c}
k \\
k_{1}, \ldots, k_{54}
\end{array}\right) \prod_{i=0}^{2} \frac{l_{i} ! m_{i} ! n_{i} !}{\left(l_{i}+m_{i}+n_{i}+3\right) !}
$$


Again, we list the first five even moments of the area of a random triangle in our tetrahedron $T_{3}$, where one point lies in $c_{3}$ :

$$
\begin{aligned}
& \mathbb{E} V_{T_{3}[3], c_{3}}^{2}=\frac{7}{2400} \approx 2.91667 \cdot 10^{-3}, \\
& \mathbb{E} V_{T_{3}[3], c_{3}}^{4}=\frac{11}{529200} \approx 2.07861 \cdot 10^{-5}, \\
& \mathbb{E} V_{T_{3}[3], c_{3}}^{6}=\frac{2839}{10973491200} \approx 2.58714 \cdot 10^{-7}, \\
& \mathbb{E} V_{T_{3}[3], c_{3}}^{8}=\frac{29419}{6224027040000} \approx 4.72668 \cdot 10^{-9}, \\
& \mathbb{E} V_{T_{3}[3], c_{3}}^{10}=\frac{4134139}{36352301290905600} \approx 1.13724 \cdot 10^{-10} .
\end{aligned}
$$

Note that the area of a triangle in $T_{3}$, where one vertex is fixed to lie in $c_{3}$, is not larger than $\sqrt{3} / 6$. Hence, we want to approximate the square root function in the interval $[0,1 / 12]$ by a polynomial

$$
Q(x)=\sum_{i=0}^{l} b_{i} x^{i}
$$

of degree $l \in \mathbb{N}$ such that $Q(x)>\sqrt{x}$ for all $x \in[0,1 / 12]$. Then we have that

$$
\mathbb{E} V_{T_{3}[3], c_{3}} \leq \mathbb{E} Q\left(V_{T_{3}[3], c_{3}}\right) \text {. }
$$

We use Lemma 2 in [4], which yields that for $m \in \mathbb{N}, l=2 m+1$ and $0<x_{0}<$ $\ldots<x_{m}$, the system of equations

$$
Q\left(x_{j}^{2}\right)=x_{j} \text { and } Q^{\prime}\left(x_{j}^{2}\right)=\frac{1}{2 x_{j}} \quad \text { for } j=0, \ldots, m
$$

determines uniquely a polynomial $Q(x)=\sum_{i=0}^{l} b_{i} x^{i}$ with the property $Q(x) \geq \sqrt{x}$ for all $x \in \mathbb{R}$. We get a lower bound on $\mathbb{E} Q\left(V_{T_{3}[3], c_{3}}\right)$ via the finite dimensional linear program

$$
\begin{gathered}
\min _{Q} \mathbb{E} Q\left(V_{T_{3}[3], c_{3}}\right)=\min _{b_{i}} \sum_{i=0}^{l} b_{i} \mathbb{E} V_{T_{3}[3], c_{3}}^{2 i} \quad \text { s.t. } Q\left(x_{\ell}^{2}\right) \geq x_{\ell}, x_{\ell} \in\left[0, \frac{\sqrt{3}}{6}\right], \\
\ell=0, \ldots, L .
\end{gathered}
$$

For $l=14$ and $L=200$ equidistant points $x_{\ell} \in\left[0, \frac{\sqrt{3}}{6}\right]$, we numerically compute via Matlab and CVX that

$$
\mathbb{E} Q\left(V_{T_{3}[3], c_{3}}\right)>0.04699
$$

and this approximation is not sufficient with consideration of (7). 
For $l=15$ and $L=1000$, we solve the above linear program, compute the interpolation nodes with the square root function numerically, and rationalize these points to

$$
\left\{x_{0}, \ldots, x_{7}\right\}=\left\{\frac{1}{45}, \frac{1}{17}, \frac{1}{11}, \frac{1}{8}, \frac{1}{6}, \frac{1}{5}, \frac{3}{13}, \frac{7}{27}\right\} .
$$

Using these points, we solve the interpolation problem from Lemma 2 in [4] and finally get a polynomial $Q_{\text {cert }}(x)=\sum_{i=0}^{15} b_{i} x^{i}$ of degree 15 with explicitly given rational coefficients $b_{0}, \ldots, b_{15}$ and the property $Q_{\text {cert }} \geq \sqrt{x}$. Inserting the even moments computed above, we see that

$$
\begin{aligned}
& \mathbb{E} V_{T_{3}[3], c_{3}} \leq \mathbb{E} Q_{\text {cert }}\left(V_{T_{3}[3], c_{3}}\right) \\
& =\frac{13643713572150037058135148101741009042438787706802976012175097880183851061858954679506499764455591316797579615557279107985989}{29065551851580796169800375743177957971396211070941311273271339321255098209543350425288830624996871957546778230784000000000000} \\
& <0.046942 \text {. }
\end{aligned}
$$

\section{Proof of Theorem 2}

\subsection{Some basic considerations}

In the first part of the proof, we use the results on the monotonicity of the moments of volumes of random full-dimensional simplices, which have already been stated in Thorem 1. It follows with Lemma 2 that, for $d \geq 2$ and $2 \leq n \leq d$, monotonicity of the map $K \rightarrow \mathbb{E} V_{K[n]}^{k}$ for $K$ ranging over all $d$-dimensional convex bodies $K$ does not hold unless $n=2$ and $k \in \mathbb{N}$ or $n=3$ and $k \in\{1,2\}$.

\subsection{Proof of Proposition 1}

Now we give a counterexample for $d \geq 2$ and $n=2$. Here we prove that random chords in a specific right-angled triangle are suitable for our purposes by giving the explicit values.

We consider the ratio $r(k):=\mathbb{E} V_{T_{2}[2], c_{2}}^{k} / \mathbb{E} V_{T_{2}[2]}^{k}$ for the triangle $T_{2}$ with vertices $(0,0),(0,1)$ and $(1,0)$ and $c_{2}=(1 / 2,1 / 2)$ the midpoint of its hypotenuse. It holds:

$$
r(k)=\frac{(k+3)(k+4)}{2^{k+3}+2^{k / 2+2}} .
$$

The ratio $r(k)$ is clearly monotonely decreasing, and $r(1)$ is smaller than 1 . Therefore, $r(k)$ is smaller than 1 for each $k \in \mathbb{N}$ and Proposition 1 holds.

\subsection{Proof of Proposition 2}

We can settle the case $n=3, k=2$ by comparing the second moments of the areas of our two different types of random triangles in a tetrahedron directly. We computed in Sect. 4: 


$$
\mathbb{E} V_{T_{3}[3], c_{3}}^{2}=\frac{7}{2400}=0.002916 \ldots<0.005625=\frac{9}{1600}=\mathbb{E} V_{T_{3}[3]}^{2}
$$

Consequently, with Lemmata 1 and 2, we have shown that the map $K \mapsto \mathbb{E} V_{K[3]}^{2}$ is not monotone if $K$ ranges over all $d$-dimensional convex bodies for $d \geq 3$.

In the same section, we have shown that

$$
\mathbb{E} V_{T_{3}[3], c_{3}}<0.046942<\mathbb{E} V_{T_{3}[3]}
$$

Hence, we also have a counterexample for $k=1, n=3$ and $d \geq 3$, and the proof of Proposition 2 as well as that of Theorem 2 are completed.

\section{Proof of Theorem 3}

Let $d \geq 3,2 \leq n \leq d$ and $k \in \mathbb{N}$. If $d>3, n \neq 3$ or $k>2$, there exists a pair of convex bodies $K, L \subseteq \mathbb{R}^{d-1}$ such that $K \subset L$, but $\mathbb{E} V_{K[n]}^{k}>\mathbb{E} V_{L[n]}^{k}$. As in the proof of Lemma 2 , for $\varepsilon>0$, we define two $d$-dimensional convex bodies by

$$
K_{\varepsilon}:=K \times[0, \varepsilon], \quad L_{\varepsilon}:=L \times[0, \varepsilon] .
$$

In the sequel, we will show that $\mathbb{E} \mathcal{V}_{K_{\varepsilon}[n]}^{k}$ and $\mathbb{E} \mathcal{V}_{L_{\varepsilon}[n]}^{k}$ converge to $\mathbb{E} V_{K[n]}^{k}$ and $\mathbb{E} V_{L[n]}^{k}$, resp., for $\varepsilon$ tending to zero. Therefore, there exists an $\varepsilon_{0}>0$ such that $\mathbb{E} \mathcal{V}_{K_{\varepsilon_{0}}[n]}^{k}>\mathbb{E} \mathcal{V}_{L_{\varepsilon_{0}}[n]}^{k}$, but $K_{\varepsilon_{0}} \subseteq L_{\varepsilon_{0}}$.

Let $X_{0}, \ldots, X_{n-1}$ be random points in bd $K_{\varepsilon}$ and

$$
N_{0}=\#\left\{X_{i} \in K \times\{0, \varepsilon\}\right\}, \quad N_{1}=\#\left\{X_{i} \in \text { bd } K \times[0, \varepsilon]\right\} .
$$

Then $N_{0}+N_{1}=n$. It holds for $0 \leq i \leq n-1$ :

$$
\begin{aligned}
\mathbb{P}\left(X_{i} \in K \times\{0, \varepsilon\}\right) & =\frac{2 \operatorname{vol}_{d-1} K}{2 \operatorname{vol}_{d-1} K+S(K) \varepsilon}, \\
\mathbb{P}\left(X_{i} \in \operatorname{bd} K \times[0, \varepsilon]\right) & =\frac{S(K) \varepsilon}{2 \operatorname{vol}_{d-1} K+S(K) \varepsilon},
\end{aligned}
$$

if $S(K)$ is the surface area of the boundary of $K$. Therefore, we have that

$$
\begin{aligned}
\mathbb{E}_{K_{\varepsilon}[n]}^{k}= & \mathbb{P}\left(N_{1}=0\right) \mathbb{E} \mathcal{V}_{K \times\{0, \varepsilon\}[n]}^{k}+\mathbb{P}\left(N_{1}>0\right) \mathbb{E}_{N_{1}>0} \mathcal{V}_{K_{\varepsilon}[n]}^{k} \\
= & \frac{2^{n} \operatorname{vol}_{d-1} K^{n}}{\left(2 \operatorname{vol}_{d-1} K+S(K) \varepsilon\right)^{n}} \mathbb{E} \mathcal{V}_{K \times\{0, \varepsilon\}[n]}^{k} \\
& +\left(1-\frac{2^{n} \operatorname{vol}_{d-1} K^{n}}{\left(2 \operatorname{vol}_{d-1} K+S(K) \varepsilon\right)^{n}}\right) \mathbb{E}_{N_{1}>0} \mathcal{V}_{K_{\varepsilon}[n]}^{k}
\end{aligned}
$$

If $\varepsilon$ tends to zero, $\mathbb{P}\left(N_{1}>0\right)$ and hence the second summand in (10) tend to zero. Now, an argument similar to that in the proof of Lemma 2 yields that $\mathbb{E} \mathcal{V}_{K \times\{0, \varepsilon\}[n]}^{k}$ 
converges to $\mathbb{E} V_{K[n]}^{k}$, and the same is true for $K$ replaced by $L$, which completes the proof.

Acknowledgements Open access funding provided by Paris Lodron University of Salzburg.

Open Access This article is distributed under the terms of the Creative Commons Attribution 4.0 International License (http://creativecommons.org/licenses/by/4.0/), which permits unrestricted use, distribution, and reproduction in any medium, provided you give appropriate credit to the original author(s) and the source, provide a link to the Creative Commons license, and indicate if changes were made.

\section{References}

1. Buchta, C., Reitzner, M.: The convex hull of random points in a tetrahedron: solution of Blaschke's problem and more general results. J. Reine Angew. Math. 536, 1-29 (2001)

2. Grant, M., Boyd, S.: CVX: Matlab Software for Disciplined Convex Programming, version 2.1., http:// cvxr.com/cvx (2014)

3. Hug, D.: Random polytopes. In: Spodarev, E. (ed.): Stochastic Geometry, Spatial Statistics and Random Fields. Lecture Notes in Mathematics 2068, pp. 205-238, Springer, Heidelberg (2013)

4. Kunis, S., Reichenwallner, B., Reitzner, M.: Monotonicity of the Sample Range of 3-D Data: Moments of Volumes of Random Tetrahedra. arXiv:1612.01893v1

5. Meckes, M.: Monotonicity of volumes of random simplices. In: Recent Trends in Convex and Discrete Geometry (2006)

6. Miles, R.E.: Isotropic random simplices. Adv. Appl. Probab. 3, 353-382 (1971)

7. Rademacher, L.: On the monotonicity of the expected volume of a random simplex. Mathematika 58, 77-91 (2012)

8. Reichenwallner, B., Reitzner, M.: On the monotonicity of the moments of volumes of random simplices. Mathematika 62, 949-958 (2016)

9. Reitzner, M.: Random polytopes. In: Kendall, W.S., Molchanov, I. (eds.) New Perspectives in Stochastic Geometry, pp. 45-76. Oxford University Press, Oxford (2010)

10. Schneider, R., Weil, W.: Stochastic and Integral Geometry. Probability and its Applications (New York). Springer, Berlin (2008)

11. Solomon, H.: Geometric Probability. Regional Conference Series in Applied Mathematics 28, SIAM, Philadelphia (1978)

12. Zinani, A.: The expected volume of a tetrahedron whose vertices are chosen at random in the interior of a cube. Monatsh. Math. 139, 341-348 (2003) 